Voix et Images

volxetimages

\title{
La Création de Gérard Bessette de Réjean Robidoux
}

\section{Paul Perron}

Volume 14, numéro 1 (39), automne 1988

France Théoret : narratrice de la subjectivité

URI : https://id.erudit.org/iderudit/200758ar

DOI : https://doi.org/10.7202/200758ar

Aller au sommaire du numéro

Éditeur(s)

Université du Québec à Montréal

ISSN

0318-9201 (imprimé)

1705-933X (numérique)

Découvrir la revue

Citer cet article

Perron, P. (1988). La Création de Gérard Bessette de Réjean Robidoux. Voix et Images, 14(1), 125-127. https://doi.org/10.7202/200758ar d'utilisation que vous pouvez consulter en ligne.

https://apropos.erudit.org/fr/usagers/politique-dutilisation/ 


\title{
La Création de Gérard Bessette ${ }^{1}$ de Réjean Robidoux
}

\author{
par Paul Perron, Université de Toronto
}

Que peut-on savoir d'un homme? Comment peut-on reconstituer, par delà les bribes qui nous parviennent, l'intelligibilité de l'œuvre d'une vie? Étrange pari à la fin du vingtième siècle que de vouloir cerner, résumer et expliciter le vécu et la production littéraire d'un homme de lettres contemporain. ([M]ais comment savoir comment être sûr)? nous dirait Omer Marin dans le Semestre.

Réjean Robidoux connaît Gérard Bessette et pratique son ouvre depuis bientôt vingt-cinq ans et la première de ses nombreuses études importantes consacrées à l'écrivain date de 1965. Aboutissement d'une longue gestation critique, l'ouvrage témoigne d'une connaissance approfondie et réelle de l'œuvre et de l'homme Bessette. D'ailleurs, Réjean Robidoux qualifie sa relation avec l'auteur d'amitié critique et le livre bénéficie à la fois d'une longue cohabitation avec l'auvre littéraire bessettienne (intégrale) (p. 10) et d'un contact personnel qui a laissé des traces dans de nombreuses lettres qui éclairent l'œuvre en question. En somme, nul n'était mieux placé pour écrire cette première monographie séminale sur la création de Bessette.

S'il est vrai, pour paraphraser Sartre, qu'il n'existe pas d'essence de l'œuvre en soi, mais que le génie de l'auteur, même réduit aux œuvres produites, n'en équivaut pas moins à l'infinité des points de vue que l'on pourrait prendre sur celle-ci et que l'on nommera son inépuisabilité, qu'en est-il du point de vue critique adopté sur l'œuvre bessettienne? Dans cette perspective, l' «Introduction» du volume s'avère révélatrice, car non seulement son auteur dévoile-t-il sa situation d'énonciation dans la mesure où, dit-il, pour lui la critique est une affaire personnelle, subjective et engagée, mais encore, puisque son étude se veut d'abord et surtout littéraire, il se place sous le signe de l'éclectisme des moyens. Et c'est justement une des principales qualités de l'étude en question, car il existe une véritable relation osmotique entre Réjean Robidoux et Gérard Bessette ainsi qu'une connaissance certaine et une maitrise admirable du champ critique de la modernité. Toutefois, la question se pose de savoir comment on peut étudier la création d'un auteur avec toutes ces méthodes éclectiques, et comment, au cours de cette étude, ces méthodes peuvent se conditionner l'une l'autre et trouver leur place respective au sein d'une démarche théorique homogène et cohérente.

La première partie du livre, intitulée "Cheminements biographiques», qui a pour tâche de nous bien faire connaître l'homme pour saisir l'œuvre, et vice versa (p. 13), comprend en plus d'un court texte liminaire, une «Chronologie de Gérard Bessette et/ou (imaginaire) d'Omer Marin» de treize pages et sept chapitres de longueur inégale qui retracent de façon plus ou moins chronologique les moments saillants de cette vie en établissant des liens avec les ouvrages produits. Ainsi, le premier chapitre, «Enfances», après avoir postulé le rapport 
étroit chez Bessette entre le biographique dans le sens très large du terme (imaginaire, faits psychiques, etc.) et l'écrit, étudie le rôle et la fonction de la configuration familiale (29-31), du père (31-32), de la mère (32), convoquant souvenirs de l'auteur, écrits (auto)biographiques, fictions, et montre comment le tout aboutit inexorablement à l'écriture. La vie éclairant les écrits et les écrits la vie. Les chapitres suivants obéissent plus ou moins au même modèle de composition où le vécu particulier, évoqué au départ, est toujours orienté vers le projet d'écriture. Post hoc ergo propter hoc? D'ailleurs, tout au cours de cette première partie, la modalisation énonciative du critique assume deux registres radicalement différents: précautionneux et hésitant lorsqu'il s'agit d'établir un lien entre la vie et l'écrit. À titre d'exemple, citons le premier paragraphe de la page 43: G. Bessette a dû continuer... On peut sans doute conjecturer... il semble avoir été ...; affirmatif... entier... confirmant toujours sur des règles. classiques... ou personnelles... les dires et jugements qu'il porte ( $($. 42) sur les ouvrages de Bessette.

Il est évident que le but de Réjean Robidoux n'est pas ici de reconstituer la vie de Bessette, mais de saisir seuls les moments pouvant éclairer le processus créateur de l'auteur. Pour ce faire, il adopte différents points de vue, certains d'inspiration historique, d'autres d'inspiration psychanalytique, et tente de restituer les composantes de l'œedipe personnel de l'auteur à l'origine de la totalité du projet littéraire (p. 67). On regrette toutefois que Réjean Robidoux n'ait pas tenté de mettre en place les médiations théoriques qui permettraient de combiner la psychanalyse et l'histoire (autant personnelle que collective). D'ailleurs, cette première partie aurait gagné en clarté si les notions avaient été mieux définies et je me demande pourquoi usage n'a pas été fait des travaux, par exemple, de Benveniste sur les tropes ou de Lacan sur l'écriture pour éclairer le projet bessettien.

La transition entre la première partie qui traite de l'homme et l'œuvre et la deuxième partie consacrée uniquement à la création s'assure par l'entremise de vingt photographies et la reproduction d'une lettre manuscrite de Gérard Bessette à Réjean Robidoux qui rejouent, dans leur matérialité même, le rituel du familial et de l'écriture évoqué dans la première partie. Cette partie charnière demanderait une analyse poussée portant sur le statut de la relation entre l'écrit et la photo qui, pour reprendre Roland Barthes, a pour fonction d'informer, représenter, faire signifier et de faire investir chez le Spectator, ce champ très vaste du désir nonchalant, qu'est le studium.

La deuxième moitié du livre qui se penche uniquement sur l'œuvre de création chez Bessette peut revendiquer un statut d'exemplarité. Réjean Robidoux passe minutieusement en revue selon leur date de parution aussi bien les Poèmes temporels de jeunesse que les nombreux écrits romanesques. Et c'est justement dans cette partie qu'il démontre l'étendue de ses connaissances de l'œuvre de Bessette et une grande sensibilité envers la chose littéraire. Il entreprend la première étude de fond des poèmes qui, en dépit des consécrations officielles de prix, ont été trop longtemps négligés par la critique. Après s'être penché sur la 
réception de l'œuvre et l'unité du recueil, Réjean Robidoux examine chaque poème d'un point de vue critique à la fois pour ce qui est de la forme et du fond afin de dégager la puissance génétique qui informe par la suite toute nouvelle et changeante manifestation d'écriture (p. 101).

Les autres chapitres examinent les premiers romans de style traditionnel: la Bagarre, le Libraire, les Pédagogues; le roman de transition: la Commensale; les romans du genre nouveau: l'Incubation, le Cycle, les Anthropoïdes; le romanbilan: le Semestre; et un demier court chapitre survole les deux ouvrages les plus récents de l'auteur: le Garden-party de Christophine et les Dires d'Omer Marin. Chacun des chapitres constitue une étude originale et approfondie de l'œuvre protéiforme de Bessette, poète et romancier. Disons pour être bref que, de par la finesse de la démarche et la maîtrise des concepts opératoires, l'analyse de Réjean Robidoux déplie le tissu narratif de chaque ouvrage, le fixe sur la page et fait apparaître ses fibres, ses nervures et ses réseaux secrets. En somme, il s'agit d'une démonstration magistrale qui montre que rien n'est simple, ni chez Bessette ni ailleurs, et que la moindre phrase écrite obéit à des règles et à des fonctionnements d'une grande complexité.

Si la réussite d'un ouvrage critique se mesure par le désir du lecteur de relire, repenser et retravailler l'œuvre objet, alors on peut dire que le livre de Réjean Robidoux est pleinement réussi et qu'il constituera un ouvrage de référence indispensable à la fois pour ce qui est de la production littéraire de Gérard Bessette et de la création littéraire en général.

1 Réjean Robidoux, la Création de Gérard Bessette, Montréal, Québec/Amérique, 1987. $208 \mathrm{p}$. 